\title{
Distinguons éthique et morale - une formation postgrade est nécessaire
}

\section{Rouven Porz}

Dr phil., dipl. biol., p.-d., responsable du service Ethique de I'Hôpital de l'lle à Berne, membre de la rédaction Ethique

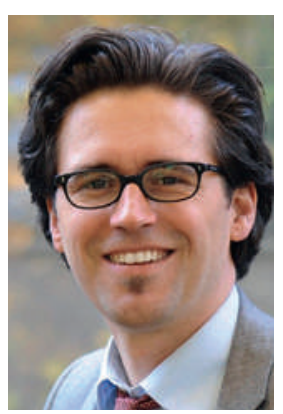

Permettez-moi d'écrire quelques lignes aujourd'hui sur mon propre métier, l'éthique. Je pense qu'en Suisse, l'éthique clinique n'est pas encore assez professionnalisée. Je parle bien de professionnalisation, pas de bureaucratisation. A mon avis, il manque en Suisse une offre de formation postgrade pour les membres des comités d'éthique clinique. ${ }^{1}$

Mais commençons par le début, avec un peu de provocation. Imaginez-vous que je travaille dans un hôpital, qu'il me semble que les médecins ont une formation psychologique trop légère et que les patients n'ont pas de suivi psychologique. Je m'adresse donc à la direction de l'hôpital: «Bonjour, j'aimerais créer une commission de psychologie. Je pense que nous en avons besoin.» Quelle est la première question qu'on va me poser? A mon avis, on me dirait quelque chose comme: «D'accord, bonne idée, mais avez-vous une formation professionnelle en psychologie? Sans formation dans ce domaine, vous ne pouvez pas créer une commission de psychologie.» Encore plus caricatural: Je m'adresse à la direction de l'hôpital. «Je suis personnellement intéressé par la chirurgie et je souhaite créer une commission de chirurgie. J'ai envie de pratiquer quelques petites interventions.» Vous pouvez imaginer quelle serait la réponse de la direction de l'hôpital, qui me prendrait probablement pour un fou. Mais pourquoi ça marche avec l'éthique? Pourquoi les membres des comités d'éthique peuvent-ils siéger sans devoir forcément justifier d'une formation dans ce domaine? Je crois connaître les raisons et il y en a plusieurs. J'en citerais deux, que je vais essayer de réfuter sans attendre.

a) Bien entendu, nous avons tous une part d'expérience éthique, je parlerais plutôt de «morale». Nous sommes tous des êtres humains, nous avons tous déjà eu mauvaise conscience, un sentiment de culpabilité, et nous avons tous été déjà amenés à prendre des décisions lourdes. Mais l'éthique est justement la discipline qui permet de réfléchir à nos propres expériences morales, de les isoler et de les replacer dans un contexte institutionnel et contemporain. Il existe pour cela des connaissances et des méthodes (qui doivent être étudiées), personne n'est obligé de partir de zéro. b) Médecins et soignants ont parfois tendance à se considérer comme ayant une expérience de l'éthique, parce qu'ils ont vécu beaucoup de situations tragiques, complexes et difficiles à trancher. C'est un fait. Mais il ne s'agit pas d'une expertise éthique, c'est une expérience morale incroyablement fructueuse et utile (voir a)). Il s'agit maintenant de la canaliser d'une nouvelle manière, et de l'utiliser avec un peu plus de recul par rapport à leurs propres valeurs. Pour ce faire, il faut adopter une certaine attitude, avec laquelle on peut aussi se familiariser au cours des formations postgrade.

\section{Je parle bien de professionnalisation, pas de bureaucratisation.}

Je vois une autre nécessité de faire de l'éthique une discipline professionnalisée: transmettre justement ces connaissances éthiques, ces méthodes éthiques et ces attitudes éthiques au personnel des hôpitaux, qui veut les aborder sous un angle "professionnel»; les spécialistes de la santé qui veulent utiliser de façon nouvelle leur propre expérience morale. Autre avantage d'une éthique clinique professionnalisée en Suisse: les comités d'éthique clinique pourraient échanger et apprendre les uns des autres, sans que chacun doive réinventer la roue à chaque fois. Il n'est tout simplement pas possible que le comité d'éthique de chaque hôpital suisse rédige ses propres instructions sur la façon de gérer les directives anticipées des patients par exemple. C'est une perte de temps et, selon moi, le signe d'un manque de professionnalisme.

Vous pensez maintenant que j'exagère. Un comité d'éthique ne peut pas provoquer des dommages aussi sévères qu'une commission de chirurgie composée de profanes. L'éthique ferait partie des sciences molles, la chirurgie des sciences dures. Je ne crois pas. Quand des décisions importantes pour l'existence, comme dans les services de soins intensifs ou les services pédiatriques, etc., sont concernées par des discussions de cas soi-disant «éthiques», dans la pire configuration, cette forme d'éthique peut faire plus de mal que ma petite intervention chirurgicale réalisée par un profane. 